\title{
Multiple stimulation parameters influence efficacy of deep brain stimulation in parkinsonian mice
}

\author{
Jonathan S. Schor ${ }^{1,2,3}$ and Alexandra B. Nelson ${ }^{1,2,3,4}$ \\ ${ }^{1}$ Neuroscience Program, ${ }^{2}$ Kavli Institute for Fundamental Neuroscience, ${ }^{3}$ Weill Institute for Neuroscience, and ${ }^{4}$ Department of Neurology, University of California, San Francisco, San Francisco, California, USA.
}

\begin{abstract}
Deep brain stimulation (DBS) is used to treat multiple neuropsychiatric disorders, including Parkinson's disease (PD). Despite widespread clinical use, its therapeutic mechanisms are unknown. Here, we developed a mouse model of subthalamic nucleus (STN) DBS for PD, to permit investigation using cell type-specific tools available in mice. We found that electrical STN DBS relieved bradykinesia, as measured by movement velocity. In addition, our model recapitulated several hallmarks of human STN DBS, including rapid onset and offset, frequency dependence, dyskinesia at higher stimulation intensity, and associations among electrode location, therapeutic benefit, and side effects. We used this model to assess whether highfrequency stimulation is necessary for effective STN DBS and whether low-frequency stimulation can be effective when paired with compensatory adjustments in other parameters. We found that low-frequency stimulation, paired with greater pulse width and amplitude, relieved bradykinesia. Moreover, a composite metric incorporating pulse width, amplitude, and frequency predicted therapeutic efficacy better than frequency alone. We found a similar relationship between this composite metric and movement speed in a retrospective analysis of human data, suggesting that correlations observed in the mouse model may extend to human patients. Together, these data establish a mouse model for elucidating mechanisms of DBS.
\end{abstract}

\section{Introduction}

Parkinson's disease (PD) is a neurodegenerative disease characterized by motor deficits, including bradykinesia (slowed movement), rigidity, and tremor. Dopamine replacement therapy relieves many motor symptoms, but is often complicated by the development of prominent motor fluctuations and involuntary movements (1). With few effective pharmacological alternatives, patients are often implanted with electrodes in basal ganglia nuclei for chronic stimulation. Deep brain stimulation of the subthalamic nucleus (STN DBS) (2) is highly effective in relieving bradykinesia, rigidity, and tremor (3). Despite several decades of clinical experience, however, the underlying mechanisms and ideal therapeutic parameters for STN DBS remain unclear (4).

The basal ganglia circuit in PD patients exhibits abnormal firing rate, pattern, and synchronization (5); STN DBS may disrupt or correct one or a number of these changes. At present, clinical practice is delivery of continuous pulsatile stimulation at high frequency. Therapeutic benefit depends on the frequency, current amplitude, and pulse width of stimulation (4). One theory postulates that high-frequency stimulation (HFS) (over $90 \mathrm{~Hz}$ ) disrupts abnormal basal ganglia activity (6), while other parameters (pulse width and current) may allow spread of stimulation through the STN. In fact, HFS provides greater relief of bradykinesia and tremor, whereas very low-frequency stimulation (LFS) (around $10 \mathrm{~Hz}$ ) can be ineffective or even potentially deleterious (7). However, a less widely held hypothesis is that any manipulation that perturbs

Conflict of interest: The authors have declared that no conflict of interest exists. Copyright: () 2019, American Society for Clinical Investigation.

Submitted: May 21, 2018; Accepted: June 11, 2019; Published: August 12, 2019.

Reference information: J Clin Invest. 2019;129(9):3833-3838.

https://doi.org/10.1172/JCl122390. neuronal firing may disrupt abnormal activity, and thus clinicians can compensate for a low setting of any one parameter (frequency, pulse width, or current) by increasing the other two; one study found the current amplitude necessary to relieve rigidity varied inversely with the pulse width (8). A better understanding of how individual parameters contribute to STN DBS efficacy would not only improve symptom management in patients, but might also identify potential therapeutic mechanisms of STN DBS.

Though electrical DBS has been demonstrated in both parkinsonian nonhuman primates $(9,10)$ and rats $(11,12)$, a mouse model, combined with the extensive mouse genetic toolbox, would permit complementary cellular and circuit investigations of DBS therapeutic mechanisms. Here, we developed a mouse model of STN DBS in hemiparkinsonian mice. Stimulation relieves bradykinesia and recapitulates a number of features of STN DBS in human patients. We used this model to investigate the relationship between stimulation parameters and therapeutic efficacy, as well as adverse effects, and found that a composite metric based on all 3 stimulation parameters predicted STN DBS efficacy. This relationship also holds in a retrospective analysis of human data, suggesting shared therapeutic features in human and mouse STN DBS. Together, our results provide a valuable tool for predicting DBS efficacy as well as a model for further investigation of STN DBS.

\section{Results and Discussion}

Parkinsonian mouse models have contributed to our understanding of both disease pathophysiology and the actions of levodopa (5). However, it is not known whether parkinsonian mice respond to electrical STN DBS in ways similar to that of patients. We identified 6 clinical features of STN DBS for PD, which we used as criteria in a mouse model of STN DBS: (i) STN DBS reduces bradykinesia; (ii) motor benefits are time locked to stimulation; (iii) relief of bra- 

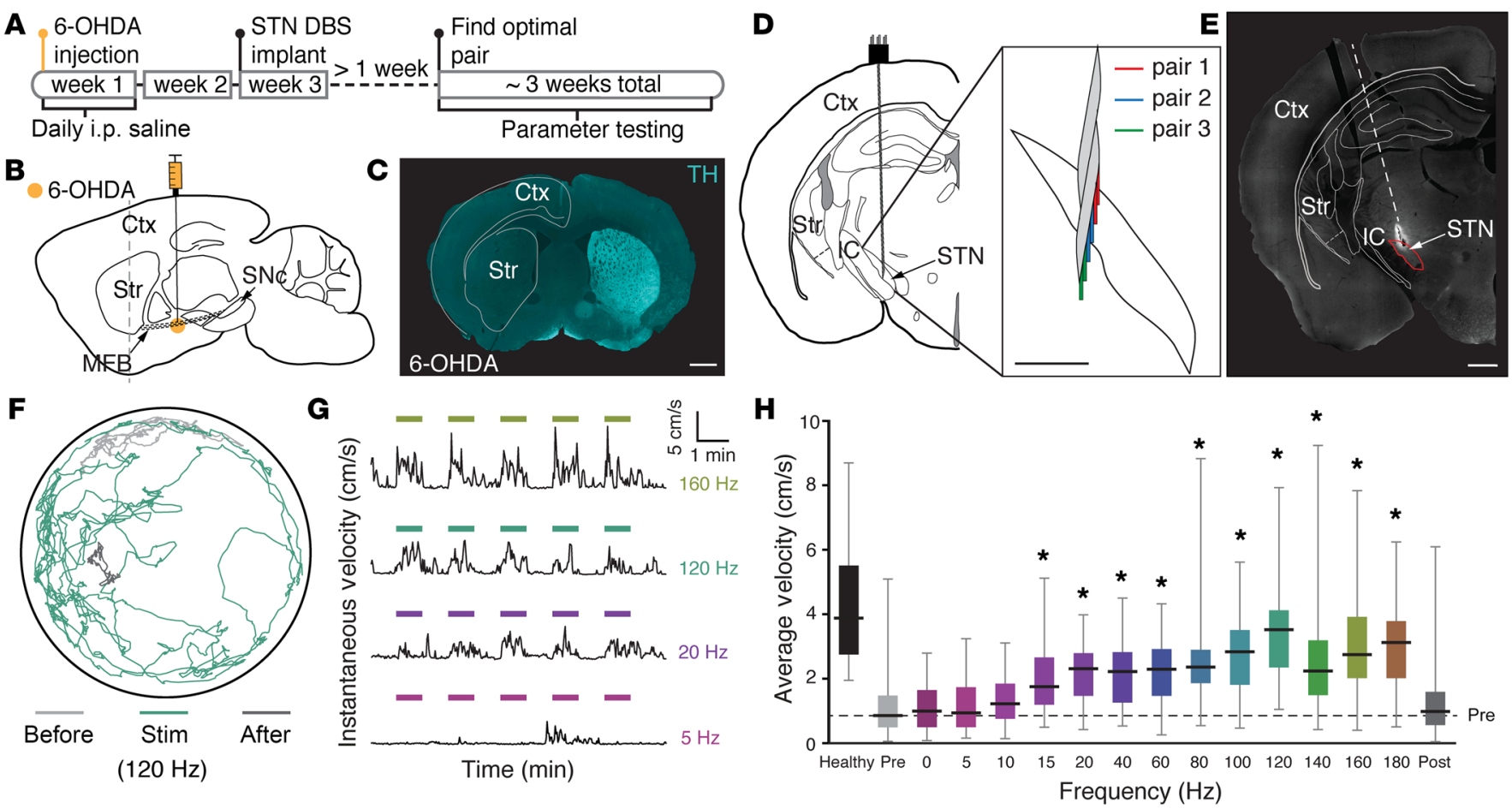

Figure 1. STN DBS alleviates bradykinesia in parkinsonian mice across a wide range of frequencies. (A) Experimental time line. (B) Sagittal schematic showing unilateral 6-OHDA medial forebrain bundle (MFB) injection. (C) Representative coronal section immunostained for tyrosine hydroxylase (TH) showing ipsilateral depletion of striatal TH. Scale bar: $750 \mu \mathrm{m}$. (D and E) Coronal schematic (D) and histological section (E) showing ipsilesional STN targeting of DBS electrode (dotted white line and terminal electrolytic lesion). Scale bars: $250 \mu \mathrm{m}$ (left panel); $750 \mu \mathrm{m}$ (right panel). (F) Representative open field movement before, during, and after $120 \mathrm{~Hz}$ STN DBS in a parkinsonian mouse (5 minutes each). (G) Representative raw velocity traces over standard 11-minute trials, consisting of 5 one-minute bouts of $5 \mathrm{~Hz}, 20 \mathrm{~Hz}, 120 \mathrm{~Hz}$, and $160 \mathrm{~Hz}$ STN DBS interleaved with 6 one-minute rest bouts. (H) Average velocity of parkinsonian mice during stimulation epochs across frequencies with constant pulse width (60 $\mu$ s) and constant current (200 $\mu \mathrm{A}$ ). Healthy refers to nonparkinsonian mice. Pre and post refer to 30 seconds before and after stimulation. Box extends from 25 th to 75 th percentile; median is indicated by horizontal line. Whiskers represent maximum and minimum values. $n=9$ healthy mice; $n=9$ parkinsonian mice. Significance determined by 1 -way repeated measures ANOVA followed by Tukey's honest significant difference test. ${ }^{*} P<0.05$ compared with prestim period. Ctx, cortex; Str, striatum; SNc, substantia nigra pars compacta; IC, internal capsule.

dykinesia is frequency dependent (13); (iv) increasing stimulation intensity (across multiple parameters) results in dyskinesia (14); (v) dorsal STN stimulation is more effective than ventral stimulation in relieving bradykinesia (15); and (vi) stimulation closer to the pyramidal tract is more likely to evoke motor contractions (16).

With these criteria in mind, we designed and implanted unilateral 6-lead electrodes (divided into 3 bipolar pairs) in the STN of adult hemiparkinsonian mice, using the unilateral 6-hydroxydopamine (6-OHDA) model (Figure 1, A-C). In these mice, parkinsonism was manifest as both ipsilesional rotational bias (Supplemental Figure 1G; supplemental material available online with this article; https://doi.org/10.1172/JCI122390DS1) and decreased movement velocity (Figure $1 \mathrm{H}$ ). We used the latter indicator as a measure of bradykinesia, though it may also incorporate other parkinsonian features, such as gait dysfunction. We constructed our 6-lead electrodes to span approximately 300-600 $\mu \mathrm{m}$ (covering the approximately $250 \mu \mathrm{m}$ vertical span of mouse STN) and implanted them in the ipsilesional STN (Figure 1, D and E, and Supplemental Figure 1, A and B). We selected the optimal electrode pair by stimulating at settings mirroring typical human DBS and DBS in rat models $(12,17)(120 \mathrm{~Hz}, 200 \mu \mathrm{A}, 60 \mu$ s with bipolar, biphasic square waves; Supplemental Figure 1C). The stimulated pair eliciting the highest average movement velocity was used for subsequent experiments (Supplemental Figure 1D). Stimulation at these DBS parameters increased movement velocity in mice (Figure 1, F and G, and Supplemental Video 1); velocity changed at short latency from onset (3.19 \pm 0.65 seconds) and offset $(0.66$ \pm 0.10 seconds) of stimulation, corroborating clinical features $\mathrm{i}$ and ii. We used 1-minute stimulation epochs to efficiently evaluate a wide array of stimulation parameter combinations, but found that longer periods of stimulation (10 minutes) produced similar improvements in movement velocity (Supplemental Figure 1E). These findings suggest that STN DBS in parkinsonian mice and PD patients share core therapeutic features.

We next explored whether STN DBS in hemiparkinsonian mice shows frequency dependence, as has been observed in humans (criterion iii) $(7,13)$. To address this question, we varied stimulation frequency while holding current amplitude and pulse width constant $(200 \mu \mathrm{A}$ and $60 \mu \mathrm{s})$. Movement velocity scaled relatively linearly, with frequency up to approximately $120 \mathrm{~Hz}$ (Figure 1H), mirroring observations in patients using hand tapping speed as the outcome measure (13). However, the range of effective frequencies was much wider (as low as $15 \mathrm{~Hz}$ ) than reported in patients. In addition to absolute velocity, STN DBS also improved other well-established metrics (17), such as percentage of time moving and relative velocity (normalized to prestim period) in a 
A
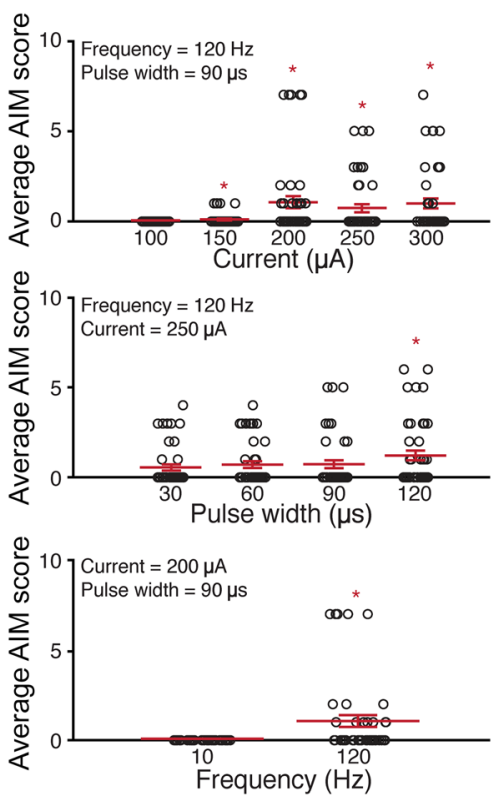

B

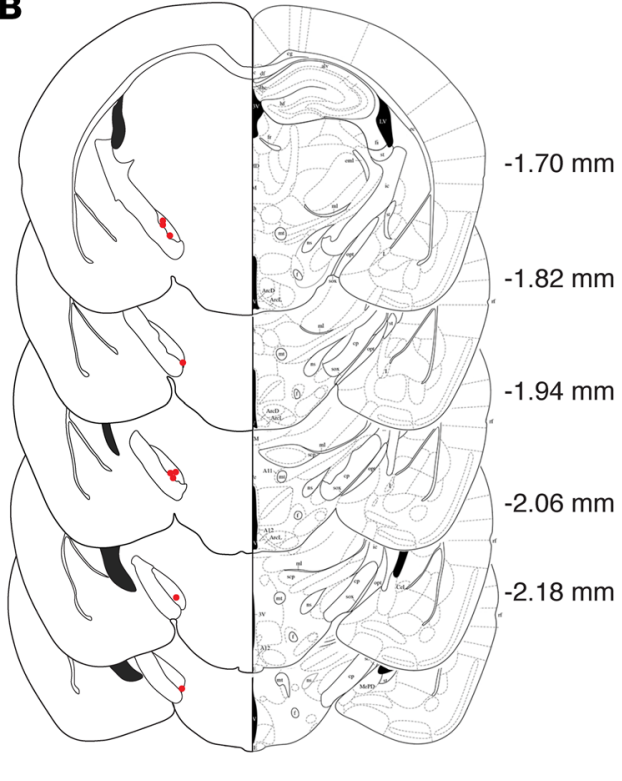

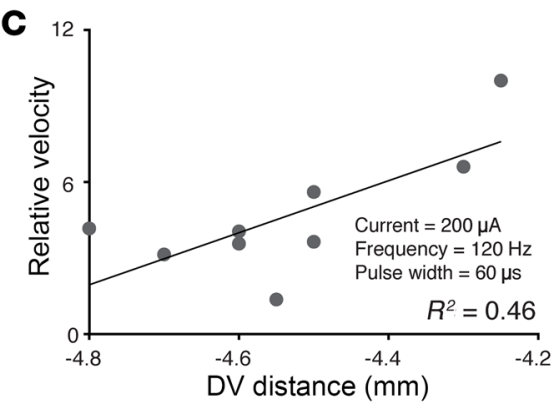

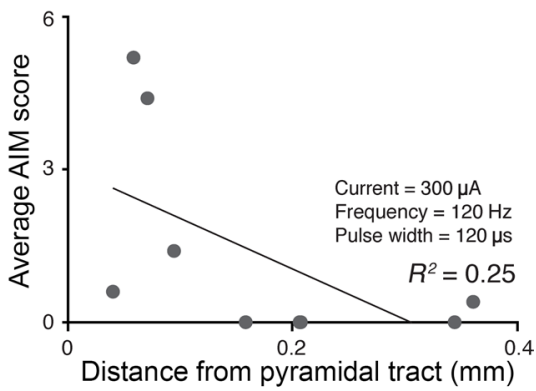

Figure 2. STN DBS in parkinsonian mice recapitulates key features of human DBS. (A) Average abnormal involuntary movement (AIM) score of parkinsonian mice during 1-minute stimulation epochs as a function of current (upper panel), pulse width (middle panel), or frequency (lower panel), holding the other 2 parameters constant ( $n=9$ mice, 5 trials per mouse per condition). (B) Stimulation sites across all mice, as determined postmortem ( $n=9$ mice; red dots). (C) Correlation between dorsoventral (DV) stimulation site and velocity increases for individual mice at standard parameters $(n=9$ mice). (D) Correlation between the stimulation site/pyramidal tract distance and average AIM score for individual mice at the parameter setting shown ( $n=9$ mice). Significance determined by 1-way repeated measures ANOVA (performed on all stimulation parameters shown in A) followed by Tukey's honest significant difference test. ${ }^{*} P<0.05$ compared with lowest stimulation setting.

frequency-dependent manner, though rotational bias was not significantly changed (Supplemental Figure 1, F-H). In humans, dyskinesia emerges as a side effect of higher frequency stimulation (14). Likewise, we found that in mice, higher frequency stimulation, as well as increased pulse width and current, evoked dyskinesia (18) (Figure 2A, criterion iv). These findings suggest that in parkinsonian mice, as in humans, both therapeutic and dyskinetic effects of stimulation relate to stimulation frequency.

To assess the relationship of stimulation location to therapeutic efficacy and dyskinesia (criteria $\mathrm{v}$ and vi), we determined the location of DBS in postmortem tissue. Following terminal anesthesia, we made electrolytic lesions using the therapeutic leads (Figure $1 \mathrm{E}$ and Figure 2B). We correlated dorsoventral location with the relative velocity of each mouse at standard stimulation settings (120 $\mathrm{Hz}, 200 \mu \mathrm{A}$, and $60 \mathrm{~s}$ ). As in humans, greater improvements in velocity were seen with more dorsal STN stimulation (Figure 2C). This may relate to targeting sensorimotor STN territories $(19,20)$ or the zona incerta, stimulation of which is also therapeutic (21, 22). We next correlated electrode distance from the pyramidal tract with the dyskinesia score in response to stimulation at $120 \mathrm{~Hz}, 300$ $\mu \mathrm{A}$, and $120 \mu$ s (parameters that reliably evoked dyskinesias in over half of mice). Again, the mouse model mirrored human data: mice with electrodes closer to the pyramidal tract had greater dyskinesia (Figure 2D). Our model fulfilled all 6 clinical criteria, recapitulating key features of human DBS and indicating it may be a useful tool in understanding STN DBS mechanisms.

A dominant theory is that HFS and LFS produce qualitatively different changes in basal ganglia activity, and thus only HFS is efficacious (7). Alternatively, any combination of frequency, cur- rent, and pulse width that sufficiently disrupts STN-basal ganglia circuit activity may be therapeutic. To test these theories using our model, we first assessed the relationship of each of the 3 variables (current, frequency, and pulse width) to movement velocity, while holding the other two constant. As we previously observed, movement velocity showed a strong linear correlation with frequency when pulse width and current were held at constant (Figure 3A). However, we also observed strong linear relationships when only varying pulse width (Figure 3B) or current (Figure 3C), suggesting each variable individually contains linear predictive value. These individual parameters can also be combined in a metric cited in the clinical DBS literature: total electrical energy delivered (TEED) (23). TEED is calculated as follows: [current ${ }^{2} \times$ frequency $\times$ pulse width]/impedance. We found that current $^{2}$, one term in this metric, was also linearly correlated with movement velocity (Figure 3D). Together, these results support the idea that many parameter combinations can provide therapeutic benefit.

While within the limited range tested, all 3 parameters correlated with therapeutic benefit, we wondered whether a combined metric might predict benefit across a wider set of parameters. If so, such a metric might prove a useful tool for clinicians. To explore this possibility, we stimulated STN using 31 different parameter combinations (Supplemental Figure 2A and Supplemental Table 1) and measured the resulting movement velocity. We collapsed each parameter combination into a single value ( $\mathrm{p}_{\mathrm{i}}^{\text {combo }}$, where the $\mathrm{i}$ subscript indicates constant-current stimulation), calculated as current ${ }^{2} \times$ frequency $\times$ pulse width. $\mathrm{p}_{\mathrm{i}}^{\text {combo }}$ is based on TEED, but we ascribe no physical meaning to this composite metric and use it only to compare different parameter combina- 

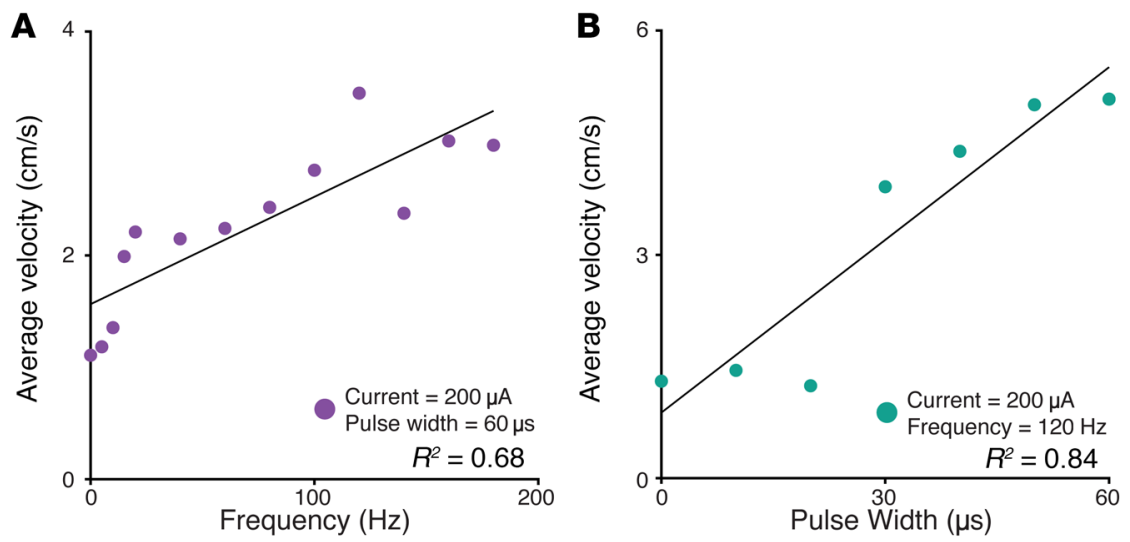

Figure 3. Effectiveness of STN DBS depends linearly on a composite stimulation parameter metric, $\mathbf{p}^{\text {combo. }}$ (A-D) In parkinsonian mice, correlation of velocity during DBS with (A) frequency ( $n=9$ mice), (B) pulse width ( $n=5$ mice), (C) current ( $n=5$ mice), or (D) current $^{2}$ ( $n=5$ mice), holding the other 2 parameters constant. (E) In parkinsonian mice, correlation of velocity with $p_{i}^{\text {combo }}$ following DBS (31 conditions, $n=9$ mice). (F) In humans, correlation of percentage of baseline tapping speed with $p_{v}^{\text {combo }}$ ( 9 conditions, $n=12$ humans) during DBS (reanalyzed from ref. 13). Each point represents an average across subjects and trials for a given condition.
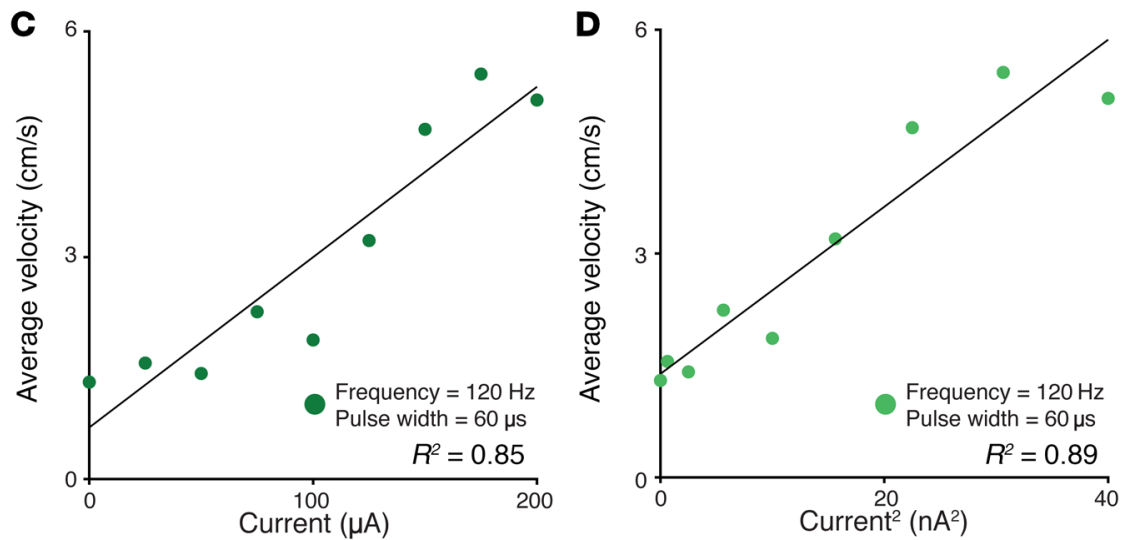

again scaled linearly with $\mathrm{p}_{\mathrm{i}}^{\text {combo }}$ (Supplemental Figure 2C). With these parameters, we also manually scored dyskinesia and found severity scaled linearly with $\mathrm{p}_{\mathrm{i}}^{\text {combo }}$ (Supplemental Figure 2D). These findings are consistent with the idea that a composite metric incorporating stimulation pulse width, amplitude, and frequency consistently explains much of the variance in both the therapeutic and dyskinetic effects of STN DBS.

We next generated a model for DBS efficacy, using the regression between stimulation parameters and movement velocity in early DBS testing. This model was based on $\mathrm{p}_{\mathrm{i}}^{\text {combo }}$ (Supplemental Figure 3, A-D) and predicts that when current and pulse width are held at levels mimicking standard human stimulation, DBS efficacy will show frequency dependence (Supplemental Figure 3, B and C). However, a more comprehensive exploration of parameter space revealed that increased pulse width and current amplitude can be used to compensate for reduced frequency, extending the range of effective DBS parameters. Similar results were observed when a model was created based on the regression from late DBS testing (Supplemental Figure 3, E and H), though due to the shallower slope of the late DBS regression, the

tions. We found that the effectiveness of DBS, as measured by movement velocity, scaled linearly with $\mathrm{p}_{\mathrm{i}}^{\text {combo }}$ (Figure $3 \mathrm{E}$ ). This relationship held across stimulation bouts when parameter combinations were grouped into $3 \mathrm{p}_{\mathrm{i}}^{\text {combo }}$ levels (Supplemental Figure 2B). These results also indicate that many different parameter combinations may be effective.

In PD patients, optimal stimulation parameters often change over the first few months of DBS use; typically, one or more parameters must be increased to maintain efficacy (24). This phenomenon may relate to changes in both the electrodes and the surrounding tissue (25). To test for this phenomenon in mice, we performed STN DBS in the same 9 mice at 50 new parameter combinations approximately 2 months later (Supplemental Figure 2A and Supplemental Table 2). Consistent with human data, mice showed less benefit from stimulation at later time points (Supplemental Figure 2C, comparing slopes of solid and dotted lines), but DBS efficacy same combination of any 3 parameters produced less therapeutic benefit than in the early model. These models not only explain previous clinical observations, but may provide valuable individualized visualizations of effective parameters for future PD patients.

To determine whether the $\mathrm{p}_{\mathrm{i}}^{\text {combo }}$ metric could be used with individual stimulation site data to predict relationships between stimulation site and therapeutic efficacy and/or dyskinesia, we calculated a metric for therapeutic efficacy. We calculated the slope of the correlation between $\mathrm{p}_{\mathrm{i}}^{\text {combo }}$ and movement velocity (Supplemental Figure 4A), representing the additional benefit derived from unit increases in $p_{i}^{\text {combo }}$. We found that electrode location along the dorsoventral axis showed a modest correlation with vel slope (Supplemental Figure 4B), consistent with human observations and our data at a single stimulation setting (Figure 2C). We next calculated a similar metric for dyskinesia: the slope of the correlation between $\mathrm{p}_{\mathrm{i}}^{\text {combo }}$ and the dyskinesia score (dysk slope), 
an indicator of tendency toward stimulation-induced dyskinesia. In line with our data from a single stimulation setting (Figure 2D), we observed that electrodes closer to the internal capsule tended to have higher dysk slopes (Supplemental Figure 4C). Finally, and perhaps most excitingly, we found no correlation between vel slope and dysk slope (Supplemental Figure 4D). These findings suggest that despite a shared correlation with $\mathrm{p}_{i}^{\text {combo }}$ improved DBS efficacy and the vulnerability to dyskinesias may be dissociable.

Optimizing patient DBS parameters is common, but extensive controlled testing is challenging in a clinical setting. Indeed, few studies have explored parameter space while measuring quantitative outcomes. However, one human study tested a subset of the parameter space (voltage, frequency, and pulse width) described here, using hand tapping as a measure of bradykinesia (13). We reanalyzed this data to determine whether a similar relationship between stimulation parameters and therapeutic effects governs STN DBS in PD patients. Based on parameter combinations tested in all 12 patients within a range equivalent to those tested in mice (Supplemental Figure 2B and Supplemental Table 3), we calculated a constant-voltage version of $\mathrm{p}_{\mathrm{i}}^{\text {combo }}\left(p_{v}^{\text {combo }}=\right.$ voltage ${ }^{2} \times$ frequency $\times$ pulse width $)$ and estimated efficacy. We found that tapping speed was strongly correlated with $\mathrm{p}_{\mathrm{v}}^{\text {combo }}$ (Figure $3 \mathrm{~F}$ ). These human results are consistent with our findings in parkinsonian mice: STN DBS efficacy for bradykinesia (as measured by locomotor velocity) scales linearly with a combined metric incorporating all 3 stimulation parameters.

Though STN DBS has provided symptomatic relief to PD patients for over 2 decades, ideal parameters for DBS, as well as their underlying therapeutic mechanisms, are still unclear. We used a set of custom-built tools to create a model of STN DBS in hemiparkinsonian mice and leveraged this model to rigorously explore the parameter space for effective DBS. We found that STN DBS is not only effective in restoring near-normal levels of locomotion in parkinsonian mice, but that it recapitulates many key features of STN DBS in PD patients. We found low-frequency DBS could be effective, provided pulse width and current amplitude were adjusted to compensate. Indeed, behavioral effectiveness depended linearly on a combination of all 3 parameters, $\mathrm{p}_{\mathrm{i}}^{\text {combo, }}$ suggesting a much larger and more predictable parameter space than previously assumed. Finally, we found that a similar metric strongly predicted relief of bradykinesia in a human DBS data set; however, due to differences in brain size, parkinsonism features, and the stimulation devices used between humans and mice, further human studies are clearly needed.

Our findings support the hypothesis that stimulation frequency does not uniquely predict DBS efficacy; indeed, multiple recent human studies also report low-frequency DBS is effective $(26,27)$. This relationship may not have been detected previously due to practical clinical barriers to systematic study of parameter space as well as outcome measures used. Utilizing a model such as ours facilitates a wider and more standardized exploration of parameter space, which can be used to generate new hypotheses for optimal human treatment.

We hope the STN DBS mouse model presented here can serve as a platform, allowing the use of the powerful mouse genetic toolbox to investigate how STN DBS modifies activity patterns in basal gangliathalamocortical circuits. These tools, such as genetically encoded calcium and voltage sensors, as well as optical and chemical manipulators of neural activity, will allow causal investigation of DBS therapeutic mechanisms in the future, complementing ongoing groundbreaking work in patients, nonhuman primates, and rat models.

\section{Methods}

For complete information, see Supplemental Methods.

Statistics. All behavioral data recorded with video tracking (Noldus Ethovision) was exported to MATLAB (MathWorks) for offline analysis. Statistical differences between stimulation parameters were assessed using 1-way repeated-measures ANOVA, followed by post hoc comparisons using Tukey's honest significant difference procedure. $P$ adjusted values reported for ANOVAs are $P$ values with the most conservative lower bound adjustment, as calculated by MATLAB. Linear correlations and adjusted $R^{2}$ values were calculated in MATLAB by fitting data to a linear model. The generalized linear model was created in MATLAB using a normal distribution.

Study approval. All the animal studies were approved by the Institutional Animal Care and Use Committee of the University of California, San Francisco.

\section{Author contributions}

JSS and ABN designed experiments. JSS built all experimental components (commutator, stimulation electrodes) and performed all experiments and analyses. JSS and ABN wrote the manuscript.

\section{Acknowledgments}

The authors would like to acknowledge P. Starr, J. Ostrem, K. Bender, D. Ron, K. Kay, A.L. Benabid, L. Ptacek, and members of the Nelson lab for providing advice and feedback on the manuscript. We thank R. Brakaj and C. Bair-Marshall for technical support. This work was supported by the National Institute of Neurological Disorders and Stroke (K08 NS081001 to ABN) and UCSF startup funds (to $\mathrm{ABN}$ ). ABN is the Richard and Shirley Cahill Endowed Chair in Parkinson's Disease Research.

Address correspondence to: Alexandra B. Nelson, UCSF Sandler Neurosciences Center, Box 0663, 675 Nelson Rising Lane, San Francisco, California 94158, USA. Phone: 415.502.7287; Email: alexandra.nelson@ucsf.edu.

\footnotetext{
1. Ahlskog JE, Muenter MD. Frequency of levodopa-related dyskinesias and motor fluctuations as estimated from the cumulative literature. Mov Disord. 2001;16(3):448-458.

2. Obeso JA, Rodríguez MC, Gorospe A, Guridi J, Alvarez L, Macias R. Surgical treatment of Parkinson's disease. Baillieres Clin Neurol. 1997;6(1):125-145.

3. Wichmann T, Delong MR. Deep brain stimula-
}

tion for neurologic and neuropsychiatric disorders. Neuron. 2006;52(1):197-204.

4. Dayal V, Limousin P, Foltynie T. Subthalamic nucleus deep brain stimulation in Parkinson's disease: the effect of varying stimulation parameters. J Parkinsons Dis. 2017;7(2):235-245.

5. McGregor MM, Nelson AB. Circuit mechanisms of Parkinson's disease. Neuron. 2019;101(6):1042-1056.
6. Chiken S, Nambu A. Disrupting neuronal transmission: mechanism of DBS? Front Syst Neurosci. 2014;8:33. doi: 10.3389/fnsys.2014.00033.

7. Eusebio A, et al. Effects of low-frequency stimulation of the subthalamic nucleus on movement in Parkinson's disease. Exp Neurol. 2008;209(1):125-130.

8. Reich MM, et al. Short pulse width widens the therapeutic window of subthalamic 
neurostimulation. Ann Clin Transl Neurol. 2015;2(4):427-432.

9. Moran A, Stein E, Tischler H, Belelovsky K, Bar-Gad I. Dynamic stereotypic responses of Basal Ganglia neurons to subthalamic nucleus high-frequency stimulation in the parkinsonian primate. Front Syst Neurosci. 2011;5:21.

10. Hashimoto T, Elder CM, Okun MS, Patrick SK, Vitek JL. Stimulation of the subthalamic nucleus changes the firing pattern of pallidal neurons. J Neurosci. 2003;23(5):1916-1923.

11. Gradinaru V, Mogri M, Thompson KR, Henderson JM, Deisseroth K. Optical deconstruction of parkinsonian neural circuitry. Science. 2009;324(5925):354-359.

12. Brocker DT, Swan BD, So RQ, Turner DA, Gross $\mathrm{RE}$, Grill WM. Optimized temporal pattern of brain stimulation designed by computational evolution. Sci Transl Med. 2017;9(371):eaah3532.

13. Moro E, et al. The impact on Parkinson's disease of electrical parameter settings in STN stimulation. Neurology. 2002;59(5):706-713.

14. Zheng Z, Li Y, Li J, Zhang Y, Zhang X, Zhuang P. Stimulation-induced dyskinesia in the early stage after subthalamic deep brain stimulation. Stereotact Funct Neurosurg. 2010;88(1):29-34.

15. Greenhouse I, Gould S, Houser M, Hicks G, Gross
J, Aron AR. Stimulation at dorsal and ventral electrode contacts targeted at the subthalamic nucleus has different effects on motor and emotion functions in Parkinson's disease. Neuropsychologia. 2011;49(3):528-534.

16. Tommasi G, et al. Pyramidal tract side effects induced by deep brain stimulation of the subthalamic nucleus. J Neurol Neurosurg Psychiatry. 2008;79(7):813-819.

17. Li Q, et al. Therapeutic deep brain stimulation in Parkinsonian rats directly influences motor cortex. Neuron. 2012;76(5):1030-1041.

18. Cenci MA, Lundblad M. Ratings of L-DOPAinduced dyskinesia in the unilateral 6-OHDA lesion model of Parkinson's disease in rats and mice. Curr Protoc Neurosci. 2007; Chapter 9:Unit 9.25.

19. Hamani C, Saint-Cyr JA, Fraser J, Kaplitt M, Lozano AM. The subthalamic nucleus in the context of movement disorders. Brain. 2004;127(Pt 1):4-20.

20. Nambu A, Takada M, Inase M, Tokuno H. Dual somatotopical representations in the primate subthalamic nucleus: evidence for ordered but reversed body-map transformations from the primary motor cortex and the supplementary motor area. J Neurosci. 1996;16(8):2671-2683.

21. Plaha P, Ben-Shlomo Y, Patel NK, Gill SS. Stimulation of the caudal zona incerta is superior to stimulation of the subthalamic nucleus in improving contralateral parkinsonism. Brain. 2006;129(Pt 7):1732-1747.

22. Blomstedt $P$, et al. Deep brain stimulation in the caudal zona incerta versus best medical treatment in patients with Parkinson's disease: a randomised blinded evaluation. J Neurol Neurosurg Psychiatry. 2018;89(7):710-716.

23. Koss AM, Alterman RL, Tagliati M, Shils JL. Calculating total electrical energy delivered by deep brain stimulation systems. Ann Neurol. 2005;58(1):168; author reply 168-168; author reply 169.

24. Wagle Shukla A, Zeilman P, Fernandez H, Bajwa JA, Mehanna R. DBS programming: an evolving approach for patients with Parkinson's disease. Parkinsons Dis. 2017;2017:8492619.

25. Satzer D, Lanctin D, Eberly LE, Abosch A. Variation in deep brain stimulation electrode impedance over years following electrode implantation. Stereotact Funct Neurosurg. 2014;92(2):94-102.

26. Khoo HM, et al. Low-frequency subthalamic nucleus stimulation in Parkinson's disease: a randomized clinical trial. Mov Disord. 2014;29(2):270-274.

27. Blumenfeld Z, et al. Sixty hertz neurostimulation amplifies subthalamic neural synchrony in Parkinson's disease. PLoS ONE. 2015;10(3):e0121067. 